\title{
Overview of D-T Results from TFTR
}

M.G. Bell, K.M. McGuire, V. Arunasalam, C.W. Barnes, ${ }^{1}$ S. Batha, ${ }^{2}$ G. Bateman, M. Beer, R.E. Bell, M. Bitter, N.L. Bretz, R. Budny, C.E. Bush, ${ }^{3}$ S.R. Cauffman, Z. Chang, ${ }^{4}$ C.S. Chang, C.Z. Cheng, D.S. Darrow, R. Dendy, ${ }^{6}$ W. Dorland, ${ }^{7}$ H. Duong, ${ }^{8}$ R. Durst, ${ }^{4}$ P.C. Efthimion, D. Ernst, ${ }^{9}$ H. Evenson, ${ }^{4}$ N. Fisch, R. Fisher, ${ }^{8}$ R.J. Fonck, ${ }^{4}$ E.D. Fredrickson, G.Y. Fu, H.P. Furth, N. Gorelenkov, ${ }^{10}$ B. Grek, L.R. Grisham, G.W. Hammett, G.R. Hanson, ${ }^{3}$ R.J. Hawryluk, W. Heidbrink, ${ }^{11}$ H.W. Herrmann, K.W. Hill, J.C. Hosea, H. Hsuan, M. Hughes, 12 R.A. Hulse, A.C. Janos, D.L. Jassby, F.C. Jobes, D.W. Johnson, L.C. Johnson, J. Kesner, ${ }^{9}$ H. Kugel, N.T. Lam, ${ }^{4}$ B. Leblanc, F.M. Levinton, ${ }^{2}$ J. Machuzak, ${ }^{9}$ R.P. Majeski, D.K. Mansfield, E. Mazzucato, M. Mauel, ${ }^{13}$ J. McChesney, ${ }^{8}$ D.C. McCune, G. McKee, ${ }^{4}$ D.M. Meade, S.S. Medley, D.R. Mikkelsen, S.V. Mirnov, ${ }^{10}$ D. Mueller, G.A. Navratil, 13 R. Nazikian, D.K. Owens, H.K. Park, W. Park, P. Parks, ${ }^{8}$ S.F. Paul, M. Petrov, ${ }^{14}$ C.K. Phillips, M. Phillips, ${ }^{12}$ S. Pitcher, ${ }^{15}$ A.T. Ramsey, M.H. Redi, G. Rewoldt, D. Roberts, ${ }^{4}$ J. Rogers, E. Ruskov, ${ }^{11}$ S.A. Sabbagh, ${ }^{13}$ M. Sasao, ${ }^{16}$ G. Schilling, J. Schivell, G.L. Schmidt, S.D. Scott, I. Semenov, ${ }^{10}$ S. Sesnic, C.H. Skinner, B.C. Stratton, J.D. Strachan, W. Stodiek, E. Synakowski, H. Takahashi, W. Tang, G. Taylor, J. Terry, ${ }^{9}$ M.E. Thompson, W. Tighe, S. Von Goeler, R. White, R.M. Wieland, J.R. Wilson, K.L. Wong, P. Woskov, ${ }^{9}$ G.A. Wurden, ${ }^{1}$ M. Yamada, K.M. Young, M.C. Zarnstorff, S.J. Zweben

Plasma Physics Laboratory, Princeton University, P.O. BoX 451, Princeton, New Jersey 08543-0451, USA

1 Los Alamos National Laboratory, Los Alamos, New Mexico

2 Fusion Physics and Technology, Torrance, California

3 Oak Ridge National Laboratory, Oak Ridge, Tennessee

4 University of Wisconsin, Madison, Wisconsin

5 Courant Institute, New York University, New York, New York

6 Culham Laboratory, Abingdon, Oxford, England

7 University of Texas, Institute for Fusion Studies, Austin, Texas

8 General Atomics, San Diego, California

9 Massachusetts Institute of Technology, Cambridge, Massachusetts

10 TRINITI, Moscow, Russia

11 University of California, Irvine, California

12 Grumman Corporation, Princeton, New Jersey

13 Columbia University, New York, New York

14 Ioffe Physical-Technical Institute, St. Petersburg, Russia

15 Canadian Fusion Fuels Technology Project, Toronto, Canada

16 National Institute of Fusion Studies, Nagoya, Japan 


\section{Abstract}

Experiments with plasmas having nearly equal concentrations of deuterium and tritium have been carried out on TFTR. To date (September 1995), the maximum fusion power has been $10.7 \mathrm{MW}$, using $39.5 \mathrm{MW}$ of neutral-beam heating, in a supershot discharge and $6.7 \mathrm{MW}$ in a high- $\beta_{\mathrm{p}}$ discharge following a current ramp-down. The fusion power density in the core of the plasma has reached $2.8 \mathrm{MWm}^{-3}$, exceeding that expected in the International Thermonuclear Experimental Reactor (ITER). The energy confinement time, $\tau_{\mathrm{E}}$, is observed to increase in $\mathrm{D}-\mathrm{T}$, relative to $\mathrm{D}$ plasmas, by $20 \%$ and the $\mathrm{n}_{\mathrm{i}}(0) \cdot \mathrm{T}_{\mathrm{i}}(0) \cdot \tau_{\mathrm{E}}$ product by $55 \%$. The improvement in thermal confinement is caused primarily by a decrease in ion heat conductivity in both supershot and limiter-H-mode discharges. Extensive lithium pellet injection increased the confinement time to $0.27 \mathrm{~s}$ and enabled higher current operation in both supershot and high- $\beta_{\mathrm{p}}$ discharges. First measurements of the confined alpha particles have been performed and found to be in good agreement with TRANSP simulations assuming classical confinement. Measurements of the alpha ash profile have been compared with simulations using particle transport coefficients from helium gas puffing experiments. The loss of energetic alpha particles to a detector at the bottom of the vessel is well described by the first-orbit loss mechanism. No loss due to alphaparticle-driven instabilities has yet been observed. ICRF heating of a D-T plasma, using the second harmonic of tritium, has been demonstrated. D-T experiments on TFTR will continue both to explore the physics underlying the ITER design and to examine some of the physics issues associated with an advanced tokamak reactor.

\section{Maximizing the fusion reactivity in TFTR}

Since December 1993, the Tokamak Fusion Test Reactor (TFTR) has been operated routinely with plasmas containing high concentrations of tritium. A variety of experiments has been conducted to study the effects of tritium on the plasma confinement and heating and the physics of the alpha-particles produced by deuterium-tritium (D-T) fusion. These TFTR experiments, which follow the JET Preliminary Tritium Experiment (PTE) in 1991 [1] with low concentrations ( 10\%) of tritium, are the first to achieve nearly optimal D-T mixtures and high fusion power densities in magnetically confined plasmas. As in the JET-PTE, injection of high-power tritium and deuterium neutral beams (NBI) has proved very successful $[2,3,4,5,6]$ for producing high $D-T$ fusion power in TFTR. The TFTR NBI sources inject almost tangentially; six of the sources inject co-parallel and six counter-parallel to the plasma current. The capability to switch each neutral beam source from deuterium to tritium operation and back on successive plasma shots has minimized the tritium consumption and has enabled careful comparisons to be made between similar D-only and D-T plasmas. The total NBI power has reached $39.5 \mathrm{MW}$ in D-T using $7 \mathrm{~T}$ and $5 \mathrm{D}$ sources (the NBI sources produce about $10 \%$ more injected power when operating in tritium). The NBI pulse has been typically 0.7 to $2.0 \mathrm{~s}$ in duration. At 5 September 1995 , a total of $2.34 \mathrm{~g}(22.5 \mathrm{kCi})$ of tritium had been introduced into the vacuum vessel by NBI and gas puffing. At that time, the total inventory of tritium in the vacuum vessel and neutral beam vacuum system following regeneration of the pumping cryo-panels (measured total tritium input minus tritium exhaust) was $0.82 \mathrm{~g}(7.9 \mathrm{kCi})$.

The highest fusion rates in TFTR for both $\mathrm{D}$-T and D-only plasmas have been obtained in "supershots" [7], characterized by very high central ion temperatures, $T_{i}(0)=20-40 \mathrm{keV} \gg \mathrm{T}_{\mathrm{e}}(0)=$ $10-12 \mathrm{keV}$, highly peaked profiles of the density and ion temperature, a broad electron temperature profile, and enhanced energy confinement. Supershots in TFTR are produced with NBI heating when the edge influxes of hydrogenic species and carbon are reduced so that the plasma core is fuelled predominantly by the injected neutrals. In addition to the enhanced confinement, this provides the advantage for D-T experiments that the central ion-species mix can be varied by changing the fraction 


\section{NOTICE}

Page(s) size did not permit electronic reproduction. Information may be purchased by the general public from the National Technical Information Service, U.S. Department of Commerce, Springfield, VA 22161 (Area Code 703-487-4650). DOE and DOE contractors may purchase information by contacting DOE's Office of Scientific and Technical Information, P.O. Box 62, Oak Ridge, TN 37831, Attn: Information Services (Area Code 423-576-8401). 

of sources injecting tritium. The edge influxes of hydrogenic species and carbon have been further reduced through the injection of solid lithium pellets ( $1-4$ pellets each containing typically $4 \times 10^{20}$ atoms) into the ohmic phase of the discharge, 1.5 - 0.5 s prior to NBI [8]. The lithium rapidly leaves the plasma and is not a significant source of plasma dilution during NBI. The use of lithium conditioning has increased the plasma current at which the supershot characteristics are obtained [9] and increased the highest energy confinement time to $0.33 \mathrm{~s}$ in a $2.3 \mathrm{MA}$ plasma with $17 \mathrm{MW}$ of tritium NBI; this confinement time is approximately 2.4 times the prediction of ITER-89P scaling [10], based on an average ion mass of 2.7. The D-T experiments have been conducted in plasmas with major radius 2.45 $-2.62 \mathrm{~m}$, minor radius $0.80-0.97 \mathrm{~m}$ having nominally circular plasma cross-section with a toroidal carbon limiter on the inboard side. The toroidal magnetic field and plasma current have been in the ranges $4.6-5.5 \mathrm{~T}$ and $0.6-2.7 \mathrm{MA}$ respectively.

In both D-T and D-only supershots, there is a strong dependence of the peak fusion rate on the total plasma energy, viz. S $\mathrm{DT} \propto \mathrm{W}_{\text {tot }}{ }^{1.9}[5,11]$. The $\beta$-limit in supershots has been found to scale similarly to the Troyon limit $[12,13]$, so that, for fixed plasma size, $W_{\text {tot,max }} \propto I_{p} B_{T}$, where $I_{p}$ is the plasma current and $\mathrm{B}_{\mathrm{T}}$ the toroidal field. A major effort has been undertaken in the past year to increase the maximum toroidal field (TF) in TFTR to exploit the improved confinement of supershots at the full NBI power available in D-T operation. After extensive analysis and review of the TF coil structure and rearrangement of the power supplies, it has proved possible to increase the TF coil current by $16 \%$ although, to date, an $8 \%$ increase has been used in plasma experiments. Coupled with a corresponding increase in the plasma current, this has increased the maximum sustainable energy in supershots by about $16 \%$ which projects to an increase of about $30 \%$ in the possible D-T fusion power.

Figure 1 shows the time evolution of the D-T fusion power and plasma stored energy for four plasmas from the experiments in May 1994 and October 1994 leading up to the shot producing the highest instantaneous power of $10.7 \pm 0.8 \mathrm{MW}$. The fusion power is measured by detectors for the $14 \mathrm{MeV}$ neutrons [14] while the plasma energy is determined from magnetic data and includes the energy in the unthermalized injected deuterons and tritons. In the experiment in May, the final shot disrupted after $0.44 \mathrm{~s}$ of NBI when it reached the $\beta$-limit at a Troyon-normalized- $\beta, \beta_{\mathrm{N}}\left(=10^{8} \cdot 2 \mu_{0}<\mathrm{p}>\mathrm{a} /\right.$ $\mathrm{B}_{\mathrm{T}} \mathrm{I}_{\mathrm{p}}$ where $\langle\mathrm{p}\rangle$ is the volume-average pressure and $\mathrm{a}$ is the plasma minor radius) of 1.9. At the higher toroidal field and plasma current available in October, TFTR was able to produce the same fusion power in a stable discharge. The shot producing the highest fusion power did suffer a minor disruption after $0.47 \mathrm{~s}$ of heating when $b_{N}$ reached 1.8. It should be noted that because the pressure profiles in supershots are highly peaked, the parameter of relevance for fusion performance, $\beta^{*}{ }_{N}\left(=10^{8} \cdot 2 \mu_{0} \sqrt{ }<p^{2}>a /\right.$ $B_{T} I_{p}$, where $\sqrt{ }\left\langle p^{2}\right\rangle$ is the root-mean-square plasma pressure) reached 2.8 in this plasma. In D-T shots with the current profile modified by ramping down the current, a fusion power of $6.7 \mathrm{MW}$ has been achieved at $\beta_{\mathrm{N}}=3.0$ and $\beta_{\mathrm{N}}^{*}=4.2[15]$.

Figure 2 shows the peak fusion power, averaged over a $40 \mathrm{~ms}$ interval, as a function of total heating power (NBI plus ohmic power; the latter is, however, negligible for $P_{\text {tot }}>10 \mathrm{MW}$ ) for supershots with NBI heating only and with more than $2 \mathrm{MW}$ tritium NBI. Plasmas with a nearly optimal D-T mixture and those with extensive lithium pellet conditioning are distinguished. A non-linear dependence of the $\mathrm{D}$-T fusion power on the heating power is apparent in these data. The highest ratio $\mathrm{Q}$ of the fusion power to the total heating power, $Q \approx 0.27$, was obtained on four shots. The shot producing $5.6 \mathrm{MW}$ with only $21 \mathrm{MW} \mathrm{NBI}$ was conditioned with four lithium pellets and achieved a total energy confinement time of $0.27 \mathrm{~s}$. The thermal plasma (electrons plus thermalized ions) accounted for about $65 \%$ of the total energy in this plasma.

The time evolution of the fusion reactivity in TFTR has been analyzed with the TRANSP code $[16,17]$. The deposition, orbit loss and slowing down of the injected $\mathrm{T}$ and $\mathrm{D}$ neutrals are calculated using the measured profiles of the electron density and the electron and ion temperatures. For the 
subset of D-T plasmas in Fig. 2 analyzed in detail by TRANSP, the model generally matches the total plasma energy within $10 \%$ and the total D-T neutron rate within $25 \%$. A further validation of the model is provided by comparing the calculated profile of the D-T neutron emission with measurements from 10 collimated neutron detectors. In TFTR, the edge recycling is dominated by deuterium since the total exposure of the limiter to tritium is small [18]. Modelling of plasmas with varying fractions of D and T injection has demonstrated that, despite the reduced level of recycling necessary for supershots, the fuelling of the core of supershots by the edge influx is quite significant [17].

In the TRANSP code, the injected deuterons and tritons are modeled as slowing classically, without radial transport, until they reach the average thermal ion energy, which can reach half of the average injection energy in good supershots. The total fusion reactivity is then the sum of components arising from thermal ions, and from reactions of the unthermalized ions with the thermal ions (beamtarget reactions) and each other (beam-beam reactions). In the plasmas producing the highest fusion power, the thermonuclear component dominates the D-T reaction rate in the dense core ( $r / a \leq 0.25$ ), although the beam-target component typically accounts for $\sim 50 \%$ and the beam-beam component $\sim 20 \%$ of the overall fusion rate. However, for these plasmas, the decomposition of the reaction rate into these three calculational components can be somewhat misleading, for two reasons. On the one hand, almost all of the tritium comes originally from the NBI, which is essential for fuelling as well as heating. On the other, in the hot plasma core, the non-Maxwellian ion distribution does not in fact increase the DT reactivity compared to that of a plasma having a locally thermalized ion distribution with the same total fuel energy and particle densities. It is the hot-ion $\left(T_{i}>T_{e}\right)$ nature of these plasmas, rather than the non-Maxwellian ion distribution, that enhances the $\mathrm{D}-\mathrm{T}$ reactivity compared to that of an isothermal $\left(T_{e}=T_{i}\right)$ plasma with the same total energy and particle densities.

The plasma with exceptional confinement produced by lithium conditioning which achieved a global $Q$ of 0.27 (Fig. 2), is calculated to have reached a central $Q$, defined as the ratio of the local fusion power to the heating power density, of 0.75 . The central fusion power densities achieved in the high-performance TFTR supershots, $1.5-2.8 \mathrm{MWm}^{-3}$, are comparable to or greater than those expected in ITER [19] at a total fusion power of $1500 \mathrm{MW}$.

\section{Confinement of fusion alpha particles}

The losses of energetic fusion alpha-particles from D-T plasmas have been measured by four energy and pitch-angle resolving particle detectors mounted near the vacuum vessel wall at $20^{\circ}, 45^{\circ}$, $60^{\circ}$ and $90^{\circ}$ below the outboard mid-plane, i.e. in the direction of the ion- $\nabla B$ drift. Scans of the plasma current have shown that in MHD-quiescent plasmas, the alpha loss rate and pitch angle distribution at the $90^{\circ}$ detector scale as expected for the prompt loss of particles born on unconfined orbits. This is shown in Fig. 3. However, for the detectors nearer the mid-plane, the first-orbit loss model does not adequately fit the data. Collisional and stochastic orbit losses in the toroidal field ripple are being investigated to explain these data.

Bursts of alpha-particle loss are sometimes correlated with MHD activity in the plasma. In general, the losses are similar to those previously reported for energetic fusion products in D-only plasmas [20] and represent only a small fraction of the alpha population. However, at major disruptions, losses of energetic alpha particles estimated to be up to $10 \%$ of the alpha population have been observed to occur in $\sim 2 \mathrm{~ms}$ during the thermal quench phase while the total current is still unperturbed. Such losses, which are observed mainly on the $90^{\circ}$ detector, could have a serious impact on first-wall components in a reactor.

The energy distribution of the alpha particles confined in the plasma has been measured for the first time in TFTR [21]. Alphas in the range 0.5-3.5 MeV have been detected through conversion to 
neutral helium by double charge-exchange in the high-density neutral cloud surrounding an ablating lithium pellet. The pellet was injected after the end of NBI, to improve its penetration, but before the alpha population had decayed. The measured spectrum is compared with the TRANSP calculation in Fig. 4. The alpha population in the lower energy range $0.1-0.6 \mathrm{MeV}$ has been detected by absolutely calibrated spectrometry of charge-exchange recombination emission [22]. The intensities of the detected signals are within a factor 2 of calculations by TRANSP.

The radial profiles of thermalized alpha particles, the helium ash, have been measured by comparing charge-exchange recombination line emission from helium in otherwise similar D-T and D-only plasmas [23]. The initial measurements have been found to be consistent with TRANSP modelling for the helium profile based on transport coefficients that had been previously determined by using external helium gas puffs [24]. With these same transport coefficients, helium ash accumulation would not quench ignition in ITER provided the density of helium at the plasma edge can be controlled.

Previous experiments in TFTR [25] and DIII-D [26] had shown that the toroidal Alfvern eigenmode (TAE), which could be driven in a reactor by the population of energetic alpha-particles, could be destabilized by the energetic ion populations created either by NBI or ICRF heating. The initial D-T experiments in TFTR, however, showed no signs of instability in the TAE frequency range and the alpha-particle loss rate remained a constant fraction of the alpha production rate as the alpha pressure increased, suggesting that deleterious collective alpha instabilities were not being excited. Theory $[27,28]$ has since shown that although TFTR achieves levels of the alpha-particle driving terms comparable to those of a reactor, the damping of the mode in supershot conditions is generally stronger than the alpha-particle drive.

\section{Confinement in D-T plasmas}

In the first D-T experiments in TFTR, it was immediately apparent that the overall energy confinement in supershots is significantly better in $\mathrm{D}-\mathrm{T}$ plasmas than in comparable $\mathrm{D}$-only plasmas. The central ion and electron temperatures also increased in the D-T plasmas. Differences in the fast-ion thermalization are expected for tritium NBI and the fusion alpha particles can provide additional heating. The effect of a possible scaling of confinement with isotopic mass has been maximized and the alpha-particle heating minimized by comparing supershots with D-only and T-only NBI.

Analysis has shown that the improvement in confinement appears to be primarily in the ion channel $[29,30]$. Figure 2.5 shows the variation of the global energy confinement time and the ion thermal diffusivity at the half minor radius for a set of plasmas with similar heating powers, currents and plasma geometry and varying fractions of T-NBI. In the highest performance supershots produced so far, the alpha particle heating of the electrons amounts to only about $1 \mathrm{MW}$ out of a total of about $10 \mathrm{MW}$ making its detection difficult. The electron temperature has been modelled in TRANSP for a quiescent $\mathrm{D}$ - $\mathrm{T}$ plasma using the electron thermal diffusivity for a $\mathrm{D}$-only reference shot. This modelling showed that if the alpha-particle heating were entirely classical, it would produce about half of the measured increase in the central electron temperature. The electron temperature rise is consistent with the combination of alpha-particle heating and scaling of the confinement with isotopic mass.

Supershots with $\mathrm{H}$-mode characteristics have been studied in both D-T and D-only plasmas $[31,32]$. The D-T H-mode plasmas have exhibited transient confinement times up to $0.24 \mathrm{~s}$, which represents an enhancement by a factor of 4 relative to the ITER-89P scaling [10] while corresponding D plasmas had enhancements of $\sim 3.2$. Across the transition to the $\mathrm{H}$-mode, the ion heat conductivity in the outer region of the plasma $(\mathrm{r} / \mathrm{a}>0.4)$ decreased by a factor of $2-3$ in the D-T plasmas whereas in $D$ plasmas the reduction factor was much lower, $<1.5$ [32]. The edge localized modes (ELMs), however, were much larger during the D-T H-modes. This suggests that ITER D-T plasmas may be more 
susceptible to giant ELMs than inferred from D-only experiments. The power threshold for the transition to an H-mode was similar in the discharges with D-only and D-T NBI. However, although the tritium content in the core of the D-T plasmas at the time of the H-mode transition was determined, from collimated neutron measurements, to be as high as $75 \%$, it was much lower in the scrape-off layer, of order $1 \%$, as determined by measurements of the $H_{\alpha}, D_{\alpha}, T_{\alpha}$ line emission [18] because the recycling influx is still predominantly deuterium from earlier exposure of the carbon limiter surface. The plasma composition at the outer boundary at the time of the $\mathrm{H}$-mode transition remains uncertain.

\section{Heating by ICRF waves in D-T plasmas}

The interactions of waves in the ion-cyclotron range of frequencies (ICRF) have also been investigated in D-T plasmas [33,34,35]. The ICRF antennas have operated well during D-T experiments and the increased radiation field, from both the D-T neutrons and the tritium $\beta$-decay, has not affected their performance.

The initial experiments combining ICRF and neutral beam heating in deuterium-tritium plasmas focused on the physics of ICRF waves. This is complicated by the possibility of multiple, spatially separated ion resonances and by potential damping on the alpha particles. In TFTR supershots positioned for good coupling of the ICRF power and with the second-harmonic tritium heating layer coincident with the Shafranov-shifted axis at $\sim 2.82 \mathrm{~m}$, the degenerate second-harmonic deuterium and fundamental hydrogen resonance layer is out of the plasma on the low field side, but the fundamental deuterium heating layer is in the plasma on the high field side at $R \approx 2.1 \mathrm{~m}$. Second-harmonic tritium heating at a power of $5.5 \mathrm{MW}$ has resulted in an increase of the central ion temperature from 26 to $36 \mathrm{keV}$ in a plasma with $23.5 \mathrm{MW}$ of neutral beam heating ( $60 \%$ in T-NBI). The electron temperature increased from 8.5 to $10.5 \mathrm{keV}$ due to both direct electron damping and minority tail heating from a $2 \%$ ${ }^{3} \mathrm{He}$ minority which was added to increase the single-pass wave damping. These results are shown in Fig. 6. Similar heating was also measured in discharges in which no ${ }^{3} \mathrm{He}$ was added. In separate experiments in which the ICRF power was modulated to increase the measurement sensitivity, the ion heating was found to be a maximum wher the second-harmonic tritium layer was on axis. These results indicate that ICRF waves can be used to heat a D-T plasma with core second-harmonic tritium damping. The measured second-harmonic tritium damping is consistent with calculations by the 2D ICRF code (SPRUCE) incorporated in TRANSP [34].

Majeski et al. [36] have suggested that an ion Bernstein wave (IBW) excited by mode-conversion from a fast wave at the $n_{\|}{ }^{2}=S$ layer in a multiple ion species plasma, such as D-T, could be used for electron heating or to drive localized electron currents. Experiments using mixed ${ }^{3} \mathrm{He}-{ }^{4} \mathrm{He}-\mathrm{D}$ plasmas have shown localized electron heating at the calculated radial position of the mode conversion surface. Up to $80 \%$ of the power is measured to be deposited on electrons at the mode conversion surface, in good agreement with numerical modelling. Central electron temperatures greater than $10 \mathrm{keV}$ have been produced with $4 \mathrm{MW}$ of RF power, the highest electron temperature achieved in TFTR in a discharge heated by RF alone. (In comparison, the highest electron temperature achieved in the hydrogen minority heating regime was $7.5 \mathrm{keV}$ with over $10 \mathrm{MW}$ of RF power.) Experiments to investigate mode conversion current drive (MCCD) and fast wave current drive (FWCD) as a means of current profile control have begun. Initial results from the FWCD experiments indicate that $70 \mathrm{kA}$ of current has been driven with $2 \mathrm{MW}$ of RF power in a plasma with a central density of $3.3 \times 10^{19} \mathrm{~m}^{-3}$, and a central electron temperature of $5 \mathrm{keV}$. With mode conversion current drive, up to $120 \mathrm{kA}$ of current has been driven on-axis in $\mathrm{D}-{ }^{4} \mathrm{He}-{ }^{3} \mathrm{He}$ plasmas with a central density of $4 \times 10^{19} \mathrm{~m}^{-3}$, and a central electron temperature of $5 \mathrm{keV}$, for a normalized current drive efficiency of $0.07 \times 10^{20} \mathrm{Am}^{-2} \mathrm{~W}^{-1}$. Off-axis currents of $100 \mathrm{kA}$ have also been driven at $\mathrm{r} / \mathrm{a} \sim 0.2$. In this latter case, the MCCD has produced 
changes in the q-profile: differences of $50 \%$ in the value of q0 are measured by the Motional Stark Effect (MSE) diagnostic between plasmas with co- and counter- MCCD [37].

An interaction has also been observed between energetic fusion products and the IBW excited by mode conversion in D-3He plasmas. In the initial experiments, the dominant observable wave interaction was believed to be with D-D fusion tritons, rather than with D-T fusion alpha particles whose population was extremely small in these plasmas. A strong enhancement of the fusion product losses detected by the probes outside the plasma was observed when the IBW was generated near the plasma axis. The loss mechanism appears to be pitch angle scattering across the passing/trapped boundary. The detectors provide some energy resolution of the escaping particles and show evidence that the fusion products are heated to approximately $1.5 \times$ their birth energy. The effect is further dependent on the phasing of the RF antennas, i.e. the direction of toroidal wave launch. For $180^{\circ}$ phasing (symmetric or non-directional launch) the effect is observed at power levels in the 3-4 MW range. For $90^{\circ}$ phasing (launch counter to the conventional current) the effect is observed with a threshold of 2-3 MW. For $270^{\circ}$ (co-parallel to the conventional current) no RF driven losses have been observed up to the power limit of $4 \mathrm{MW}$.

In a subsequent experiment, a small tritium gas puff was added to the D-3 He plasma with IBW heating. When the mode-conversion layer was close to the cyclotron resonance layer for alpha particles, there was a further increase in the measured fusion-product loss rate, suggesting that the D-T alpha particles were also interacting with the waves.

\section{Summary}

In one and a half years of experiments, TFTR has explored a wide range of physics issues in plasmas with high concentrations of tritium and achieved good progress in fusion power production. Routine operation and maintenance of the facility has been performed in the D-T environment. TFTR has been operated at and beyond its original specifications in magnetic field and neutral beam heating power during these experiments. The diagnostics have operated extremely well and a large amount of analysis has already been done to guide future experiments.

In general, deuterium-tritium plasmas show improved characteristics compared to similar deuterium plasmas. The benefits for fusion power production of operating in a regime with $T_{i}>T_{e}$ and a highly peaked pressure profile to maximize the reactivity for $a$ fixed $b$ have also been clearly demonstrated in TFTR. Increasing the toroidal magnetic field has produced a significant increase in the achievable fusion power, emphasizing that the peak, rather than the average, achievable plasma pressure is the relevant issue for fusion experiments.

\section{Acknowledgments}

We wish to thank the entire staff of the TFTR Project for their unstinting efforts in support of these experiments. We thank Drs. R.C. Davidson and P. Rutherford for their support and encouragement. This work is supported by U.S. Department of Energy Contract DE-AC02-76-CH03073.

\section{References}

[1] JET TEAM, Nucl. Fusion 32 (1992) 187.

[2] STRACHAN, J.D. et al., Phys. Rev, Lett. 72 (1994) 3526.

[3] HAWRYLUK, R.J. et al., Phys. Rev. Lett. 72 (1994) 3530.

[4] HAWRYLUK, R.J. et al., to appear in Plasma Physics and Controlled Nuclear Fusion Research 
1994 (Proc. 15th Int. Conf., Seville, Spain, Sep.1994) Paper A-1-I-1.

[5] BELL, M.G. et al., to appear in Plasma Physics and Controlled Nuclear Fusion Research 1994 (Proc. 15th Int. Conf., Seville, Spain, Sep. 1994) Paper A-2-I-1.

[6] McGUIRE, K.M. et al., Phys. Plasmas 2 (1995) 2176.

[7] STRACHAN, J.D. et al., Phys. Rev. Lett. 58 (1987) 1004 .

[8] SNIPES, J.A. et al., J. Nucl. Materials 196-198 (1992) 686.

[9] MANSFIELD, D., et al., "Enhanced D-T Supershot Performance at High Current Using Extensive Lithium Conditioning in TFTR", submitted to Phys. Plasmas.

[10] YUSHMANOV, P. et al., Nucl. Fusion 30 (1990) 1999.

[11] BELL, M.G. et al., Plasma Physics and Controlled Nuclear Fusion Research 1988 (Proc. 12th Int. Conf., Nice, France, 12-19 October, 1988) (IAEA, Vienna, 1989) Vol. 1, p. 27.

[12] TROYON, F. et al., Plasma Phys. Control. Fusion 26 (1984) 209.

[13] FREDRICKSON, E.D. et al., to appear in Plasma Physics and Controlled Nuclear Fusion Research 1994 (Proc. 15th Int. Conf., Seville, Spain, Sep. 1994) Paper A-2-II-5.

[14] JOHNSON, L.C. et al., Rev. Sci. Instrum. 66 (1995) 894.

[15] SABBAGH, S.A. et al., to appear in Plasma Physics and Controlled Nuclear Fusion Research 1994 (Proc. 15th Int. Conf., Seville, Spain, Sep. 1994) Paper A-5-I-6.

[16] BUDNY, R.V. et al., Nucl. Fusion 32 (1992) 429.

[17] BUDNY, R.V, Nucl. Fusion 34 (1994) 1247.

[18] SKINNER, C.H. et al., Nucl. Fusion 35 (1995) 143.

[19] ITER Plasma Design Description Document (Draft), March 1995 (ITER Joint Central Team, San Diego, CA, USA)

[20] ZWEBEN, S.J. et al., Phys. Plasmas 1 (1994) 1469.

[21] FISHER, R.K. et al., Phys. Rev. Lett. 75 (1995) 846.

[22] McKEE, G.R. et al., Phys. Rev. Lett. 75 (1995) 649.

[23] SYNAKOWSKI, E. et al., "Measurements of the Production and Transport of Helium Ash on the TFTR Tokamak", submitted to Phys. Rev. Lett.

[24] EFTHIMION, P.C. et al., to appear in Plasma Physics and Controlled Nuclear Fusion Research 1994 (Proc. 15th Int. Conf., Seville, Spain, Sep. 1994) Paper A-2-II-6.

[25] WONG, K.-L., FONCK, R.J., PAUL, S.F., Phys. Rev. Lett. 66 (1994) 1874.

[26] HEIDBRINK, W.W., et al., Nucl. Fusion 31 (1991) 1635.

[27] CHENG, C.Z. et al., to appear in Plasma Physics and Controlled Nuclear Fusion Research 1994 (Proc. 15th Int. Conf., Seville, Spain, Sep. 1994) Paper D-3-III-2.

[28] SPONG, D. et al., to appear in Plasma Physics and Controlled Nuclear Fusion Research 1994 (Proc. 15th Int. Conf., Seville, Spain, Sep. 1994) Paper D-P-II-3.

[29] ZARNSTORFF, M.C. et al., to appear in Plasma Physics and Controlled Nuclear Fusion Research 1994 (Proc. 15th Int. Conf., Seville, Spain, Sep. 1994) Paper A-2-I-2

[30] SCOTT, S.D. et al., Phys. Plasmas 2 (1995) 2299.

[31] BUSH, C.E. et al., Proc. 21st EPS Conference on Controlled Fusion and Plasma Physics (Montpellier, 27 June - 1 July 1994) Vol.18B, Part I (Europ. Phys. Soc., 1994) 354.

[32] BUSH, C.E. et al., Phys. Plasmas 2 (1995) 2366. 
[33] TAYLOR, G. et al., to appear in Plasma Physics and Controlled Nuclear Fusion Research 1994 (Proc. 15th Int. Conf., Seville, Spain, Sep. 1994) Paper A-3-I-3. PHILLIPS, C.K. et al., Phys. Plasmas 2 (1995) 2427.

MAJESKI, R. et al. to appear in Plasma Physics and Controlled Nuclear Fusion Research 1994 (Proc. 15th Int. Conf., Seville, Spain, Sep. 1994) Paper A-3-I-4. MAJESKI, R., PHILLIPS, C. K., WILSON, J. R., Phys. Rev. Lett. 73 (1994) 2204. submitted to Phys. Rev. Lett.

\title{
DISCLAIMER
}

\begin{abstract}
This report was prepared as an account of work sponsored by an agency of the United States Government. Neither the United States Government nor any agency thereof, nor any of their employees, makes any warranty, express or implied, or assumes any legal liability or responsibility for the accuracy, completeness, or usefulness of any information, apparatus, product, or process disclosed, or represents that its use would not infringe privately owned rights. Reference herein to any specific commercial product, process, or service by trade name, trademark, manufacturer, or otherwise does not necessarily constitute or imply its endorsement, recommendation, or favoring by the United States Government or any agency thereof. The views and opinions of authors expressed herein do not necessarily state or reflect those of the United States Government or any agency thereof.
\end{abstract}




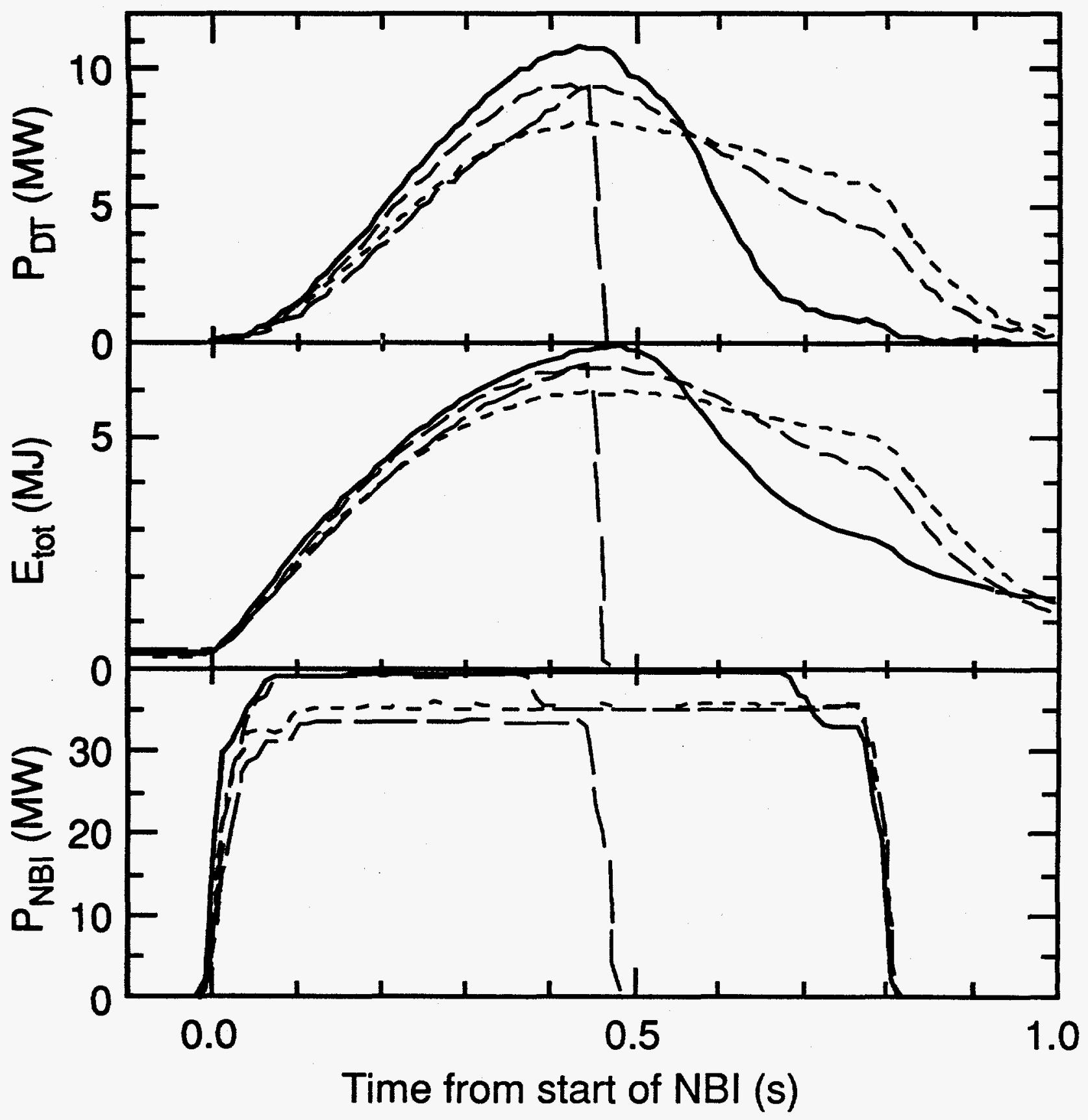

Fig. 1 Evolution of the NBI power, plasma stored energy and fusion power for the four discharges producing the highest powers. For the three non-disruptive shots, the major radius was $2.52 \mathrm{~m}$, minor radius $0.87 \mathrm{~m}$, toroidal magnetic field $5.5 \mathrm{~T}$ and the plasma current 2.7MA. For the shot which disrupted, the toroidal magnetic field was 5.1T and the plasma current 2.5MA. 


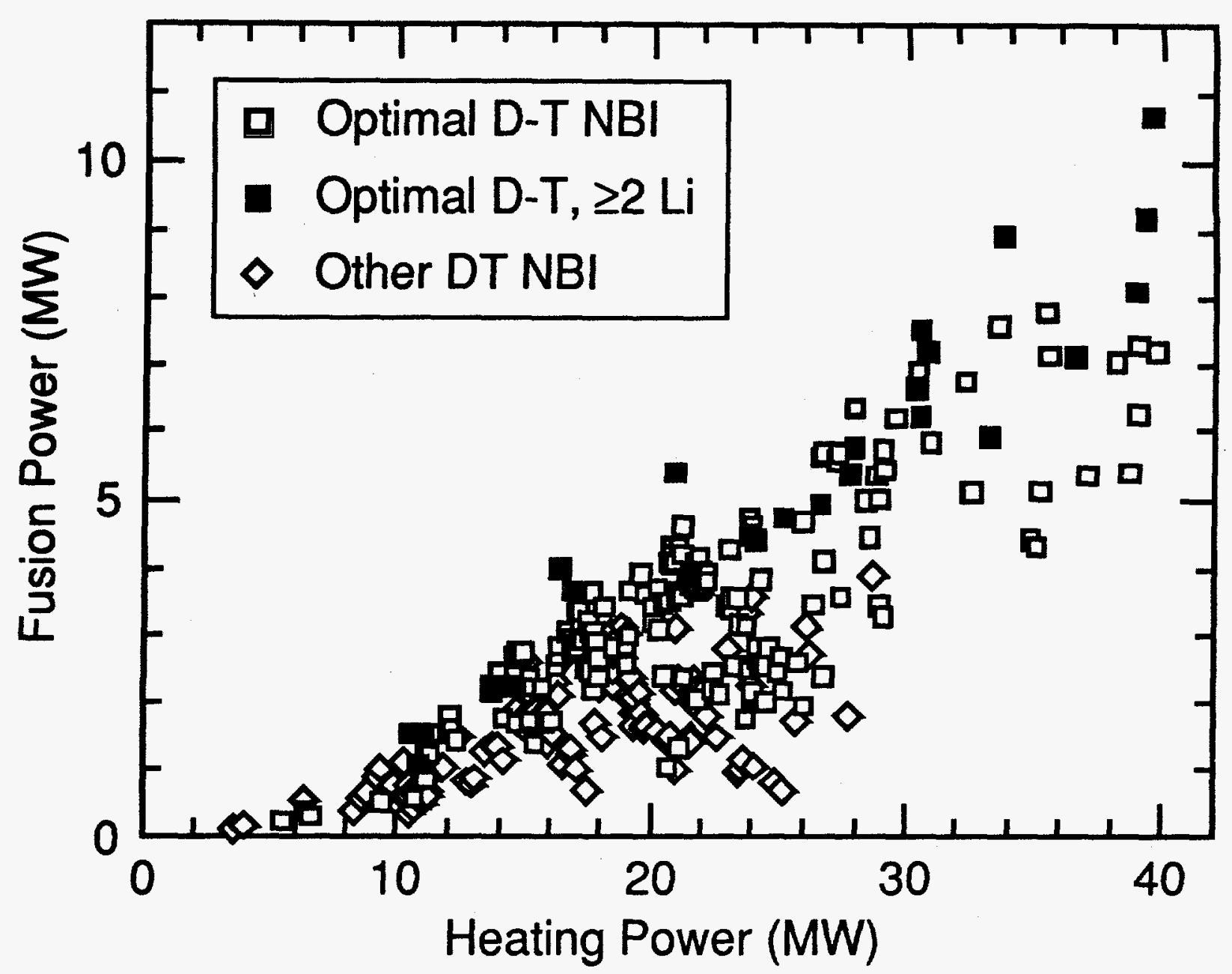

Fig. 2 Dependence of the peak DT fusion power output on the total heating power (NBI plus ohmic). The data are for NBI heated supershots with at least one source injecting tritium. Shots with nearly optimal tritium fraction, $0.4<\mathrm{P}_{\mathrm{T}-\mathrm{NBI}} / \mathrm{P}_{\mathrm{NBI}}<0.8$, are distinguished. For a given heating power, increased fusion output is made possible through conditioning the limiter by injecting multiple lithium pellets before the NBI pulse. 


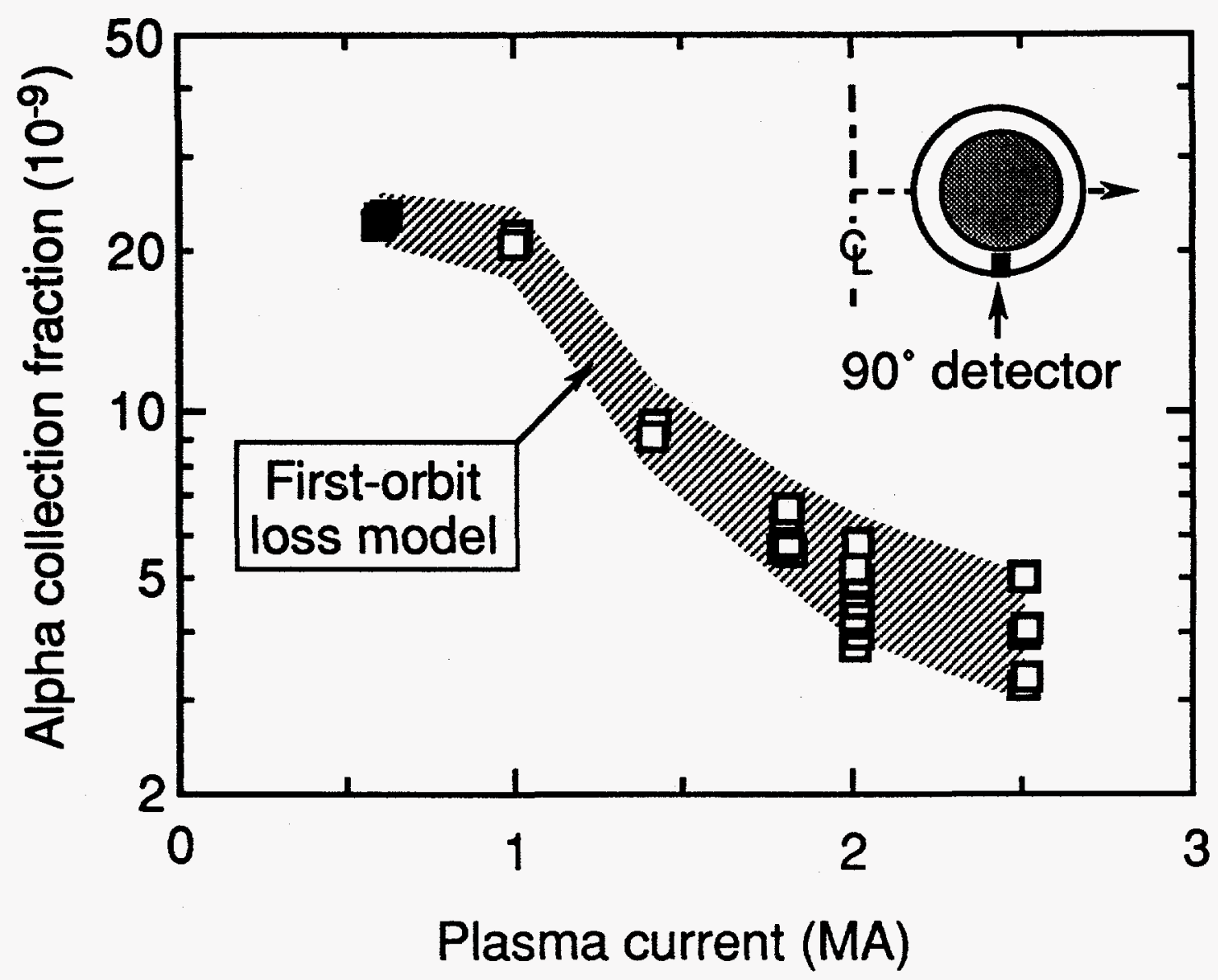

Fig.3. Dependence of the loss rate of energetic alpha-particles on the plasma current. The location of the detector is indicated in the inset. The shaded region shows the loss rate calculated for first-orbit losses. The data were normalized to the calculation at 0.6MA (solid points) where all trapped alpha-particles are lost. 


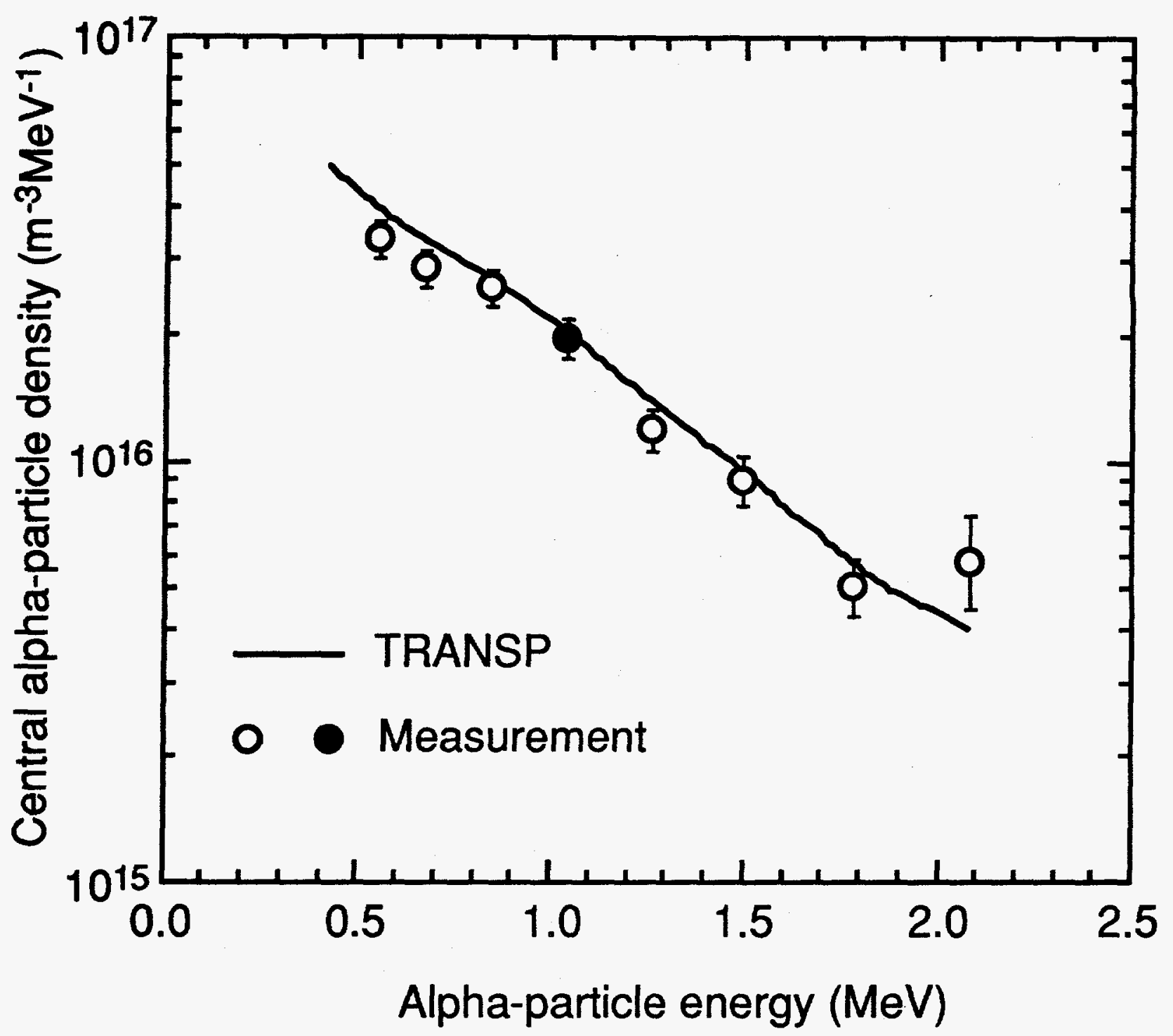

Fig. 4 Alpha-particle energy distribution at the center of a D-T plasma $0.2 \mathrm{~s}$ after the end of the NBI. The spectrum is determined by energy analysis of helium neutrals produced by double charge-exchange in the neutral cloud surrounding an ablating lithium pellet. The measurements are normalized to the TRANSP calculation at the solid point. 


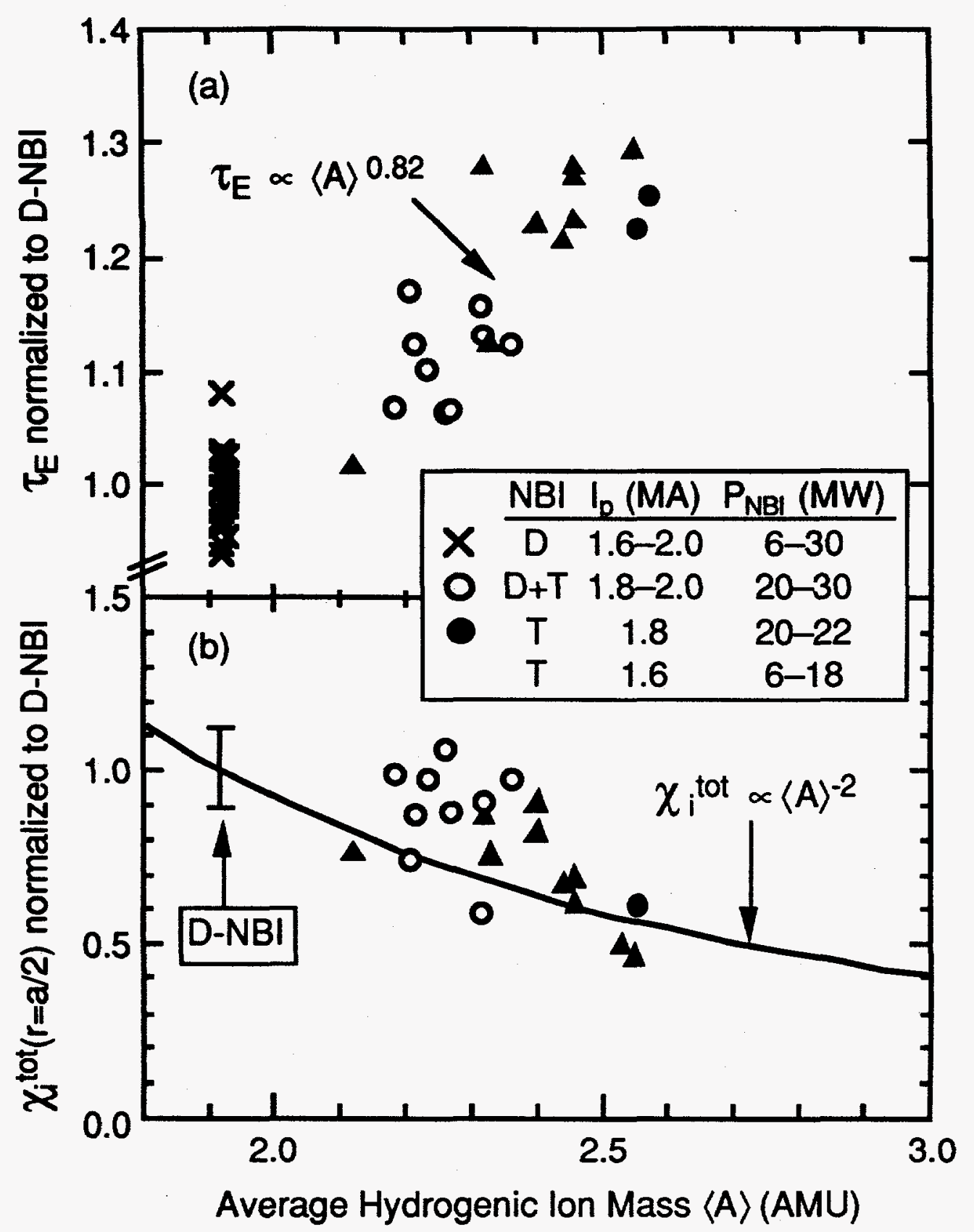

Fig. 5 Variation of (a) the global energy confinement and (b) the inferred total ion thermal diffusivity at the half minor radius with the average ion mass. Data are for supershots with varying fractions of $D$ and $T$ NBI. 

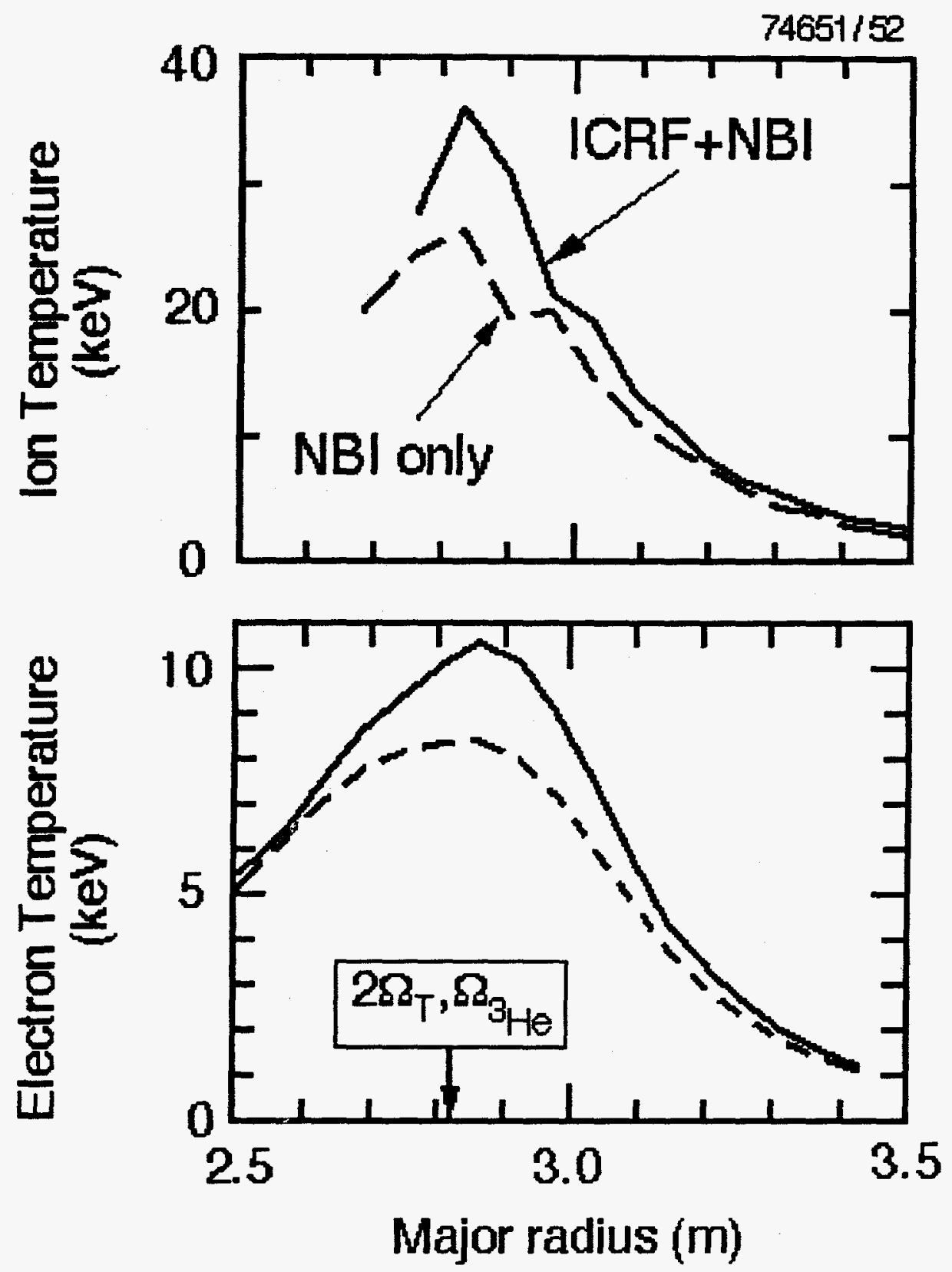

Fig. 6 Profiles of (a) the ion and (b) the electron temperature for supe rshot plasmas he ated by 13.5 MW T- and 10.0 MW D- NBI, with and without $5.5 \mathrm{MW}$ of ICRF heating. To inc rease the single-pass ICRF absorption $2 \%$ ${ }^{3} \mathrm{He}$ was added. The conditions were chosen to place the second-harmonic-tritium/fundamental-3He resonance layer on axis. In addition to the increase in temperature. the D-T reaction rate increased by about $10 \%$ with the ICRF heating. 
